\title{
Diamond- $\alpha$ Jensen's Inequality on Time Scales
}

\author{
Moulay Rchid Sidi Ammi, Rui A. C. Ferreira, and Delfim F. M. Torres
}

Department of Mathematics, University of Aveiro, 3810-193 Aveiro, Portugal

Correspondence should be addressed to Delfim F. M. Torres, delfim@ua.pt

Received 12 December 2007; Revised 10 February 2008; Accepted 7 April 2008

Recommended by Martin Bohner

The theory and applications of dynamic derivatives on time scales have recently received considerable attention. The primary purpose of this paper is to give basic properties of diamond- $\alpha$ derivatives which are a linear combination of delta and nabla dynamic derivatives on time scales. We prove a generalized version of Jensen's inequality on time scales via the diamond- $\alpha$ integral and present some corollaries, including Hölder's and Minkowski's diamond- $\alpha$ integral inequalities.

Copyright ( 2008 Moulay Rchid Sidi Ammi et al. This is an open access article distributed under the Creative Commons Attribution License, which permits unrestricted use, distribution, and reproduction in any medium, provided the original work is properly cited.

\section{Introduction}

Jensen's inequality is of great interest in the theory of differential and difference equations, as well as other areas of mathematics. The original Jensen's inequality can be stated as follows.

Theorem 1.1 (see[1]). If $g \in C([a, b],(c, d))$ and $f \in C((c, d), \mathbb{R})$ is convex, then

$$
f\left(\frac{\int_{a}^{b} g(s) d s}{b-a}\right) \leq \frac{\int_{a}^{b} f(g(s)) d s}{b-a} .
$$

Jensen's inequality on time scales via $\Delta$-integral has been recently obtained by Agarwal, Bohner, and Peterson.

Theorem 1.2 (see[2]). If $g \in C_{\mathrm{rd}}([a, b],(c, d))$ and $f \in C((c, d), \mathbb{R})$ is convex, then

$$
f\left(\frac{\int_{a}^{b} g(s) \Delta s}{b-a}\right) \leq \frac{\int_{a}^{b} f(g(s)) \Delta s}{b-a} .
$$

Under similar hypotheses, we may replace the $\Delta$-integral by the $\nabla$-integral and get a completely analogous result [3]. The aim of this paper is to extend Jensen's inequality to an arbitrary time scale via the diamond- $\alpha$ integral [4]. 
There have been recent developments of the theory and applications of dynamic derivatives on time scales. From the theoretical point of view, the study provides a unification and extension of traditional differential and difference equations. Moreover, it is a crucial tool in many computational and numerical applications. Based on the well-known $\Delta$ (delta) and $\nabla$ (nabla) dynamic derivatives, a combined dynamic derivative, the so-called $\nabla_{\alpha}$ (diamond- $\alpha$ ) dynamic derivative, was introduced as a linear combination of $\Delta$ and $\nabla$ dynamic derivatives on time scales [4]. The diamond- $\alpha$ derivative reduces to the $\Delta$ derivative for $\alpha=1$ and to the $\nabla$ derivative for $\alpha=0$. On the other hand, it represents a "weighted dynamic derivative" on any uniformly discrete time scale when $\alpha=1 / 2$. We refer the reader to [4-6] for an account of the calculus associated with the diamond- $\alpha$ dynamic derivatives.

The paper is organized as follows. In Section 2, we briefly give the basic definitions and theorems of time scales as introduced in Hilger's thesis [7] (see also [8,9]). In Section 3, we present our main results which are generalizations of Jensen's inequality on time scales. Some examples and applications are given in Section 4.

\section{Preliminaries}

A time scale $\mathbb{T}$ is an arbitrary nonempty closed subset of real numbers. The calculus of time scales was initiated by Hilger in his Ph.D. thesis [7] in order to unify discrete and continuous analysis. Let $\mathbb{T}$ be a time scale. $\mathbb{T}$ has the topology that inherits from the real numbers with the standard topology. For $t \in \mathbb{T}$, we define the forward jump operator $\sigma: \mathbb{T} \rightarrow \mathbb{T}$ by $\sigma(t)=\inf \{s \in$ $\mathbb{T}: s>t\}$, and the backward jump operator $\rho: \mathbb{T} \rightarrow \mathbb{T}$ by $\rho(t)=\sup \{s \in \mathbb{T}: s<t\}$.

If $\sigma(t)>t$, we say that $t$ is right-scattered, while if $\rho(t)<t$, we say that $t$ is leftscattered. Points that are simultaneously right-scattered and left-scattered are called isolated. If $\sigma(t)=t$, then $t$ is called right-dense, and if $\rho(t)=t$, then $t$ is called left-dense. Points that are simultaneously right-dense and left-dense are called dense. Let $t \in \mathbb{T}$, then two mappings $\mu, v: \mathbb{T} \rightarrow[0,+\infty)$ are defined as follows: $\mu(t):=\sigma(t)-t, v(t):=t-\rho(t)$.

We introduce the sets $\mathbb{T}^{k}, \mathbb{T}_{k}$, and $\mathbb{T}_{k}^{k}$, which are derived from the time scale $\mathbb{T}$, as follows. If $\mathbb{T}$ has a left-scattered maximum $t_{1}$, then $\mathbb{T}^{k}=\mathbb{T}-\left\{t_{1}\right\}$, otherwise $\mathbb{T}^{k}=\mathbb{T}$. If $\mathbb{T}$ has a rightscattered minimum $t_{2}$, then $\mathbb{T}_{k}=\mathbb{T}-\left\{t_{2}\right\}$, otherwise $\mathbb{T}_{k}=\mathbb{T}$. Finally, we define $\mathbb{T}_{k}^{k}=\mathbb{T}^{k} \cap \mathbb{T}_{k}$.

Throughout the text, we will denote a time scales interval by

$$
[a, b]_{\mathbb{T}}=\{t \in \mathbb{T}: a \leq t \leq b\}, \quad \text { with } a, b \in \mathbb{T} .
$$

Let $f: \mathbb{T} \rightarrow \mathbb{R}$ be a real function on a time scale $\mathbb{T}$. Then, for $t \in \mathbb{T}^{k}$, we define $f^{\Delta}(t)$ to be the number, if one exists, such that for all $\epsilon>0$ there is a neighborhood $U$ of $t$ such that for all $s \in U:$

$$
\left|f(\sigma(t))-f(s)-f^{\Delta}(t)(\sigma(t)-s)\right| \leq \epsilon|\sigma(t)-s| .
$$

We say that $f$ is delta differentiable on $\mathbb{T}^{k}$, provided $f^{\Delta}(t)$ exists for all $t \in \mathbb{T}^{k}$. Similarly, for $t \in \mathbb{T}_{k}$, we define $f^{\nabla}(t)$ to be the number value, if one exists, such that for all $\epsilon>0$, there is a neighborhood $V$ of $t$ such that for all $s \in V$ :

$$
\left|f(\rho(t))-f(s)-f^{\nabla}(t)(\rho(t)-s)\right| \leq \epsilon|\rho(t)-s| .
$$

We say that $f$ is nabla differentiable on $\mathbb{T}_{k}$, provided that $f^{\nabla}(t)$ exists for all $t \in \mathbb{T}_{k}$.

Given a function $f: \mathbb{T} \rightarrow \mathbb{R}$, then we define $f^{\sigma}: \mathbb{T} \rightarrow \mathbb{R}$ by $f^{\sigma}(t)=f(\sigma(t))$ for all $t \in \mathbb{T}$, that is, $f^{\sigma}=f \circ \sigma$. We define $f^{\rho}: \mathbb{T} \rightarrow \mathbb{R}$ by $f^{\rho}(t)=f(\rho(t))$ for all $t \in \mathbb{T}$, that is, $f^{\rho}=f \circ \rho$. The following properties hold for all $t \in \mathbb{T}^{k}$. 
(i) If $f$ is delta differentiable at $t$, then $f$ is continuous at $t$.

(ii) If $f$ is continuous at $t$ and $t$ is right-scattered, then $f$ is delta differentiable at $t$ with $f^{\Delta}(t)=\left(f^{\sigma}(t)-f(t)\right) / \mu(t)$.

(iii) If $f$ is right-dense, then $f$ is delta differentiable at $t$ if and only if the $\operatorname{limit}_{\lim } \rightarrow t(f(t)-$ $f(s)) /(t-s)$ exists as a finite number. In this case, $f^{\Delta}(t)=\lim _{s \rightarrow t}(f(t)-f(s)) /(t-s)$.

(iv) If $f$ is delta differentiable at $t$, then $f^{\sigma}(t)=f(t)+\mu(t) f^{\Delta}(t)$.

Similarly, given a function $f: \mathbb{T} \rightarrow \mathbb{R}$, the following is true for all $t \in \mathbb{T}_{k}$.

(a) If $f$ is nabla differentiable at $t$, then $f$ is continuous at $t$.

(b) If $f$ is continuous at $t$ and $t$ is left-scattered, then $f$ is nabla differentiable at $t$ with $f^{\nabla}(t)=\left(f(t)-f^{\rho}(t)\right) / v(t)$.

(c) If $f$ is left-dense, then $f$ is nabla differentiable at $t$ if and only if the limit $\lim _{s \rightarrow t}(f(t)-f(s)) /(t-s)$ exists as a finite number. In this case, $f^{\nabla}(t)=$ $\lim _{s \rightarrow t}(f(t)-f(s)) /(t-s)$.

(d) If $f$ is nabla differentiable at $t$, then $f^{\rho}(t)=f(t)-v(t) f^{\nabla}(t)$.

A function $f: \mathbb{T} \rightarrow \mathbb{R}$ is called rd-continuous, provided it is continuous at all right-dense points in $\mathbb{T}$ and its left-sided limits exist at all left-dense points in $\mathbb{T}$.

A function $f: \mathbb{T} \rightarrow \mathbb{R}$ is called ld-continuous, provided it is continuous at all left-dense points in $\mathbb{T}$ and its right-sided limits exist finite at all right-dense points in $\mathbb{T}$.

A function $F: \mathbb{T} \rightarrow \mathbb{R}$ is called a delta antiderivative of $f: \mathbb{T} \rightarrow \mathbb{R}$, provided $F^{\Delta}(t)=f(t)$ holds for all $t \in \mathbb{T}^{k}$. Then the delta integral of $f$ is defined by $\int_{a}^{b} f(t) \Delta t=F(b)-F(a)$.

A function $G: \mathbb{T} \rightarrow \mathbb{R}$ is called a nabla antiderivative of $g: \mathbb{T} \rightarrow \mathbb{R}$, provided $G^{\nabla}(t)=g(t)$ holds for all $t \in \mathbb{T}_{k}$. Then the nabla integral of $g$ is defined by $\int_{a}^{b} g(t) \nabla t=G(b)-G(a)$.

For more details on time scales, we refer the reader to [10-16]. Now, we briefly introduce the diamond- $\alpha$ dynamic derivative and the diamond- $\alpha$ integral $[4,17]$.

Let $\mathbb{T}$ be a time scale, and $t, s \in \mathbb{T}$. Following [17], we define $\mu_{\mathrm{ts}}=\sigma(t)-s, \eta_{\mathrm{ts}}=\rho(t)-s$, and $f \diamond_{\alpha}(t)$ to be the value, if one exists, such that for all $\epsilon>0$ there is a neighborhood $U$ of $t$ such that for all $s \in U$,

$$
\left|\alpha\left[f^{\sigma}(t)-f(s)\right] \eta_{\mathrm{ts}}+(1-\alpha)\left[f^{\rho}(t)-f(s)\right] \mu_{\mathrm{ts}}-f^{\diamond_{\alpha}}(t) \mu_{\mathrm{ts}} \eta_{\mathrm{ts}}\right|<\epsilon\left|\mu_{\mathrm{ts}} \eta_{\mathrm{ts}}\right|
$$

A function $f$ is said diamond- $\alpha$ differentiable on $\mathbb{T}_{k}^{k}$, provided $f^{\diamond_{\alpha}}(t)$ exists for all $t \in \mathbb{T}_{k}^{k}$. Let $0 \leq \alpha \leq 1$. If $f(t)$ is differentiable on $t \in \mathbb{T}_{k}^{k}$ both in the delta and nabla senses, then $f$ is diamond- $\alpha$ differentiable at $t$ and the dynamic derivative $f^{\diamond_{\alpha}}(t)$ is given by

$$
f^{\diamond_{\alpha}}(t)=\alpha f^{\Delta}(t)+(1-\alpha) f^{\nabla}(t)
$$

(see [17, Theorem 3.2]). Equality (2.5) is the definition of $f^{\diamond_{\alpha}}(t)$ found in [4]. The diamond- $\alpha$ derivative reduces to the standard $\Delta$ derivative for $\alpha=1$, or the standard $\nabla$ derivative for $\alpha=0$. On the other hand, it represents a "weighted dynamic derivative" for $\alpha \in(0,1)$. Furthermore, the combined dynamic derivative offers a centralized derivative formula on any uniformly discrete time scale $\mathbb{T}$ when $\alpha=1 / 2$. 
Let $f, g: \mathbb{T} \rightarrow \mathbb{R}$ be diamond- $\alpha$ differentiable at $t \in \mathbb{T}_{k}^{k}$. Then, (cf. [4, Theorem 2.3])

(i) $f+g: \mathbb{T} \rightarrow \mathbb{R}$ is diamond- $\alpha$ differentiable at $t \in \mathbb{T}_{k}^{k}$ with

$$
(f+g)^{\diamond_{\alpha}}(t)=(f)^{\diamond_{\alpha}}(t)+(g)^{\diamond_{\alpha}}(t)
$$

(ii) For any constant $c, c f: \mathbb{T} \rightarrow \mathbb{R}$ is diamond- $\alpha$ differentiable at $t \in \mathbb{T}_{k}^{k}$ with

$$
(c f)^{\diamond_{\alpha}}(t)=c(f)^{\diamond_{\alpha}}(t)
$$

(iii) $f g: \mathbb{T} \rightarrow \mathbb{R}$ is diamond- $\alpha$ differentiable at $t \in \mathbb{T}_{k}^{k}$ with

$$
(f g)^{\diamond_{\alpha}}(t)=(f)^{\diamond_{\alpha}}(t) g(t)+\alpha f^{\sigma}(t)(g)^{\Delta}(t)+(1-\alpha) f^{\rho}(t)(g)^{\nabla}(t)
$$

Let $a, t \in \mathbb{T}$, and $h: \mathbb{T} \rightarrow \mathbb{R}$. Then the diamond- $\alpha$ integral of $h$ from $a$ to $t$ is defined by

$$
\int_{a}^{t} h(\tau) \diamond_{\alpha} \tau=\alpha \int_{a}^{t} h(\tau) \Delta \tau+(1-\alpha) \int_{a}^{t} h(\tau) \nabla \tau, \quad 0 \leq \alpha \leq 1,
$$

provided that there exist delta and nabla integrals of $h$ on $\mathbb{T}$. It is clear that the diamond$\alpha$ integral of $h$ exists when $h$ is a continuous function. We may notice that the $\nabla_{\alpha}$-combined derivative is not a dynamic derivative for the absence of its antiderivative [17, Section 4]. Moreover, in general, we do not have

$$
\left(\int_{a}^{t} h(\tau) \diamond_{\alpha} \tau\right)^{\nabla_{\alpha}}=h(t), \quad t \in \mathbb{T} .
$$

Example 2.1. Let $\mathbb{T}=\{0,1,2\}, a=0$, and $h(\tau)=\tau^{2}, \tau \in \mathbb{T}$. It is a simple exercise to see that

$$
\left.\left(\int_{0}^{t} h(\tau) \diamond_{\alpha} \tau\right)^{\diamond_{\alpha}}\right|_{t=1}=h(1)+2 \alpha(1-\alpha)
$$

so that (2.10) holds only when $\nabla_{\alpha}=\nabla$ or $\diamond_{\alpha}=\Delta$.

Let $a, b, t \in \mathbb{T}, c \in \mathbb{R}$. Then, (cf. [4, Theorem 3.7])

(a) $\int_{a}^{t}(f(\tau)+g(\tau)) \diamond_{\alpha} \tau=\int_{a}^{t} f(\tau) \diamond_{\alpha} \tau+\int_{a}^{t} g(\tau) \diamond_{\alpha} \tau$;

(b) $\int_{a}^{t} c f(\tau) \diamond_{\alpha} \tau=c \int_{a}^{t} f(\tau) \diamond_{\alpha} \tau$;

(c) $\int_{a}^{t} f(\tau) \diamond_{\alpha} \tau=\int_{a}^{b} f(\tau) \diamond_{\alpha} \tau+\int_{b}^{t} f(\tau) \diamond_{\alpha} \tau$.

Next lemma provides some straightforward, but useful results for what follows.

Lemma 2.2. Assume that $f$ and $g$ are continuous functions on $[a, b]_{\mathbb{T}}$.

(1) If $f(t) \geq 0$ for all $t \in[a, b]_{\mathbb{T}}$, then $\int_{a}^{b} f(t) \diamond_{\alpha} t \geq 0$.

(2) If $f(t) \leq g(t)$ for all $t \in[a, b]_{\mathbb{T}}$, then $\int_{a}^{b} f(t) \diamond_{\alpha} t \leq \int_{a}^{b} g(t) \diamond_{\alpha} t$.

(3) If $f(t) \geq 0$ for all $t \in[a, b]_{\mathbb{T}}$, then $f(t)=0$ if and only if $\int_{a}^{b} f(t) \diamond_{\alpha} t=0$. 
Proof. Let $f(t)$ and $g(t)$ be continuous functions on $[a, b]_{\mathbb{T}}$.

(1) Since $f(t) \geq 0$ for all $t \in[a, b]_{\mathbb{T}}$, we know (see $[15,16]$ ) that $\int_{a}^{b} f(t) \Delta t \geq 0$ and $\int_{a}^{b} f(t) \nabla t \geq 0$. Since $\alpha \in[0,1]$, the result follows.

(2) Let $h(t)=g(t)-f(t)$. Then $\int_{a}^{b} h(t) \diamond_{\alpha} t \geq 0$ and the result follows from properties (a) and (b) above.

(3) If $f(t)=0$ for all $t \in[a, b]_{\mathbb{T}}$, the result is immediate. Suppose now that there exists $t_{0} \in[a, b]_{\mathbb{T}}$ such that $f\left(t_{0}\right)>0$. It is easy to see that at least one of the integrals $\int_{a}^{b} f(t) \Delta t$ or $\int_{a}^{b} f(t) \nabla t$ is strictly positive. Then we have the contradiction $\int_{a}^{b} f(t) \diamond_{\alpha} t>0$.

\section{Main results}

We now prove Jensen's diamond- $\alpha$ integral inequalities.

Theorem 3.1 (Jensen's inequality). Let $\mathbb{T}$ be a time scale, $a, b \in \mathbb{T}$ with $a<b$, and $c, d \in \mathbb{R}$. If $g \in C\left([a, b]_{\mathbb{T}},(c, d)\right)$ and $f \in C((c, d), \mathbb{R})$ is convex, then

$$
f\left(\frac{\int_{a}^{b} g(s) \nabla_{\alpha} s}{b-a}\right) \leq \frac{\int_{a}^{b} f(g(s)) \diamond_{\alpha} s}{b-a} .
$$

Remark 3.2. In the particular case $\alpha=1$, (3.1) reduces to that of Theorem 1.2. If $\mathbb{T}=\mathbb{R}$, then Theorem 3.1 gives the classical Jensen's inequality, that is, Theorem 1.1 . However, if $\mathbb{T}=\mathbb{Z}$ and $f(x)=-\ln (x)$, then one gets the well-known arithmetic-mean geometric-mean inequality (3.18).

Proof. Since $f$ is convex, we have

$$
\begin{aligned}
f\left(\frac{\int_{a}^{b} g(s) \nabla_{\alpha} s}{b-a}\right) & =f\left(\frac{\alpha}{b-a} \int_{a}^{b} g(s) \Delta s+\frac{1-\alpha}{b-a} \int_{a}^{b} g(s) \nabla s\right) \\
& \leq \alpha f\left(\frac{1}{b-a} \int_{a}^{b} g(s) \Delta s\right)+(1-\alpha) f\left(\frac{1}{b-a} \int_{a}^{b} g(s) \nabla s\right) .
\end{aligned}
$$

Using now Jensen's inequality on time scales (see Theorem 1.2), we get

$$
\begin{aligned}
f\left(\frac{\int_{a}^{b} g(s) \nabla_{\alpha} s}{b-a}\right) & \leq \frac{\alpha}{b-a} \int_{a}^{b} f(g(s)) \Delta s+\frac{1-\alpha}{b-a} \int_{a}^{b} f(g(s)) \nabla s \\
& =\frac{1}{b-a}\left(\alpha \int_{a}^{b} f(g(s)) \Delta s+(1-\alpha) \int_{a}^{b} f(g(s)) \nabla s\right) \\
& =\frac{1}{b-a} \int_{a}^{b} f(g(s)) \nabla_{\alpha} s .
\end{aligned}
$$

Now, we give an extended Jensen's inequality on time scales via the diamond- $\alpha$ integral.

Theorem 3.3 (Generalized Jensen's inequality). Let $\mathbb{T}$ be a time scale, $a, b \in \mathbb{T}$ with $a<b, c, d \in \mathbb{R}$, $g \in C\left([a, b]_{\mathbb{T}},(c, d)\right)$, and $h \in C\left([a, b]_{\mathbb{T}}, \mathbb{R}\right)$ with

$$
\int_{a}^{b}|h(s)| \nabla_{\alpha} s>0 .
$$


If $f \in C((c, d), \mathbb{R})$ is convex, then

$$
f\left(\frac{\int_{a}^{b}|h(s)| g(s) \nabla_{\alpha} s}{\int_{a}^{b}|h(s)| \nabla_{\alpha} s}\right) \leq \frac{\int_{a}^{b}|h(s)| f(g(s)) \nabla_{\alpha} s}{\int_{a}^{b}|h(s)| \nabla_{\alpha} s} .
$$

Remark 3.4. Theorem 3.3 is the same as [3, Theorem 3.17]. However, we prove Theorem 3.3 using a different approach than that proposed in [3]: in [3], it is stated that such result follows from the analog nabla inequality. As we have seen, diamond-alpha integrals have different properties than those of delta or nabla integrals (cf. Example 2.1). On the other hand, there is an inconsistency in [3]: a very simple example showing this fact is given below in Remark 3.10.

Remark 3.5. In the particular case $h=1$, Theorem 3.3 reduces to Theorem 3.1.

Remark 3.6. If $f$ is strictly convex, the inequality sign " $\leq$ " in (3.5) can be replaced by " $<$ ". Similar result to Theorem 3.3 holds if one changes the condition " $f$ is convex" to " $f$ is concave," by replacing the inequality sign " $\leq$ " in (3.5) by " $\geq$ ".

Proof. Since $f$ is convex, it follows, for example, from [18, Exercise 3.42C], that for $t \in(c, d)$ there exists $a_{t} \in \mathbb{R}$ such that

$$
a_{t}(x-t) \leq f(x)-f(t) \quad \forall x \in(c, d)
$$

Setting

$$
t=\frac{\int_{a}^{b}|h(s)| g(s) \diamond_{\alpha} s}{\int_{a}^{b}|h(s)| \diamond_{\alpha} s}
$$

then using (3.6) and item (2) of Lemma 2.2, we get

$$
\begin{aligned}
\int_{a}^{b}|h(s)| f(g(s)) \nabla_{\alpha} s-\left(\int_{a}^{b}|h(s)| \nabla_{\alpha} s\right) f\left(\frac{\int_{a}^{b}|h(s)| g(s) \diamond_{\alpha} s}{\int_{a}^{b}|h(s)| \nabla_{\alpha} s}\right) \\
\quad=\int_{a}^{b}|h(s)| f(g(s)) \diamond_{\alpha} s-\left(\int_{a}^{b}|h(s)| \nabla_{\alpha} s\right) f(t) \\
\quad=\int_{a}^{b}|h(s)|(f(g(s))-f(t)) \diamond_{\alpha} s \\
\geq a_{t}\left(\int_{a}^{b}|h(s)|(g(s)-t)\right) \diamond_{\alpha} s \\
=a_{t}\left(\int_{a}^{b}|h(s)| g(s) \diamond_{\alpha} s-t \int_{a}^{b}|h(s)| \nabla_{\alpha} s\right) \\
=a_{t}\left(\int_{a}^{b}|h(s)| g(s) \diamond_{\alpha} s-\int_{a}^{b}|h(s)| g(s) \diamond_{\alpha} s\right)=0 .
\end{aligned}
$$

This leads to the desired inequality. 
Remark 3.7. The proof of Theorem 3.3 follows closely the proof of the classical Jensen's inequality (see, e.g., [18, Problem 3.42]) and the proof of Jensen's inequality on time scales $[2]$.

We have the following corollaries.

Corollary $3.8(\mathbb{T}=\mathbb{R})$. Let $g, h:[a, b] \rightarrow \mathbb{R}$ be continuous functions with $g([a, b]) \subseteq(c, d)$ and $\int_{a}^{b}|h(x)| d x>0$. If $f \in C((c, d), \mathbb{R})$ is convex, then

$$
f\left(\frac{\int_{a}^{b}|h(x)| g(x) d x}{\int_{a}^{b}|h(x)| d x}\right) \leq \frac{\int_{a}^{b}|h(x)| f(g(x)) d x}{\int_{a}^{b}|h(x)| d x} .
$$

Corollary $3.9(\mathbb{T}=\mathbb{Z})$. Given a convex function $f$, we have for any $x_{1}, \ldots, x_{n} \in \mathbb{R}$ and $c_{1}, \ldots, c_{n} \in \mathbb{R}$ with $\sum_{k=1}^{n}\left|c_{k}\right|>0$ :

$$
f\left(\frac{\sum_{k=1}^{n}\left|c_{k}\right| x_{k}}{\sum_{k=1}^{n}\left|c_{k}\right|}\right) \leq \frac{\sum_{k=1}^{n}\left|c_{k}\right| f\left(x_{k}\right)}{\sum_{k=1}^{n}\left|c_{k}\right|} .
$$

Remark 3.10. Corollary 3.9 coincides with [19, Corollary 2.4] and [3, Corollary 3.12] if one substitutes all the $\left|c_{k}\right|^{\prime}$ s in Corollary 3.9 by $c_{k}$ and we restrict ourselves to integer values of $x_{i}$ and $c_{i}, i=1, \ldots, n$. Let $\mathbb{T}=\mathbb{Z}, a=1$, and $b=3$, so that $[a, b]_{\mathbb{T}}$ denotes the set $\{1,2,3\}$ and $n=3$. For the data $f(x)=x^{2}, c_{1}=1, c_{2}=5, c_{3}=-3, x_{1}=1, x_{2}=1$, and $x_{3}=2$ one has $A=\sum_{k=1}^{3} c_{k}=3>0$, and $B=\sum_{k=1}^{3} c_{k} x_{k}=0$. Thus $D=f(B / A)=f(0)=0$. On the other hand, $f\left(x_{1}\right)=1, f\left(x_{2}\right)=1$, and $f\left(x_{3}\right)=4$. Therefore, $C=\sum_{k=1}^{3} c_{k} f\left(x_{k}\right)=-6$. We have $E=C / A=-2$ and $D>E$, that is, $f\left(\sum_{k=1}^{n} c_{k} x_{k} / \sum_{k=1}^{n} c_{k}\right)>\sum_{k=1}^{n} c_{k} f\left(x_{k}\right) / \sum_{k=1}^{n} c_{k}$. Inequality (3.10) gives the truism $16 / 9 \leq 2$.

\section{Particular cases}

(i) Let $g(t)>0$ on $[a, b]_{\mathbb{T}}$ and $f(t)=t^{\beta}$ on $(0,+\infty)$. One can see that $f$ is convex on $(0,+\infty)$ for $\beta<0$ or $\beta>1$, and $f$ is concave on $(0,+\infty)$ for $\beta \in(0,1)$. Then

$$
\begin{aligned}
& \left(\frac{\int_{a}^{b}|h(s)| g(s) \diamond_{\alpha} s}{\int_{a}^{b}|h(s)| \diamond_{\alpha} s}\right)^{\beta} \leq \frac{\int_{a}^{b}|h(s)| g^{\beta}(s) \diamond_{\alpha} s}{\int_{a}^{b}|h(s)| \diamond_{\alpha} s}, \quad \text { if } \beta<0 \text { or } \beta>1 ; \\
& \left(\frac{\int_{a}^{b}|h(s)| g(s) \diamond_{\alpha} s}{\int_{a}^{b}|h(s)| \diamond_{\alpha} s}\right)^{\beta} \geq \frac{\int_{a}^{b}|h(s)| g^{\beta}(s) \diamond_{\alpha} s}{\int_{a}^{b}|h(s)| \diamond_{\alpha} s}, \quad \text { if } \beta \in(0,1) .
\end{aligned}
$$

(ii) Let $g(t)>0$ on $[a, b]_{\mathbb{T}}$ and $f(t)=\ln (t)$ on $(0,+\infty)$. One can also see that $f$ is concave on $(0,+\infty)$. It follows that

$$
\ln \left(\frac{\int_{a}^{b}|h(s)| g(s) \diamond_{\alpha} s}{\int_{a}^{b}|h(s)| \diamond_{\alpha} s}\right) \geq \frac{\int_{a}^{b}|h(s)| \ln (g(s)) \diamond_{\alpha} s}{\int_{a}^{b}|h(s)| \diamond_{\alpha} s} .
$$


(iii) Let $h=1$, then

$$
\ln \left(\frac{\int_{a}^{b} g(s) \diamond_{\alpha} s}{b-a}\right) \geq \frac{\int_{a}^{b} \ln (g(s)) \diamond_{\alpha} s}{b-a}
$$

(iv) Let $\mathbb{T}=\mathbb{R}, g:[0,1] \rightarrow(0, \infty)$ and $h(t)=1$. Applying Theorem 3.3 with the convex and continuous function $f=-\ln$ on $(0, \infty), a=0$, and $b=1$, we get

$$
\ln \int_{0}^{1} g(s) d s \geq \int_{0}^{1} \ln (g(s)) d s
$$

Then

$$
\int_{0}^{1} g(s) d s \geq \exp \left(\int_{0}^{1} \ln (g(s)) d s\right)
$$

(v) Let $\mathbb{T}=\mathbb{Z}$ and $n \in \mathbb{N}$. Fix $a=1, b=n+1$ and consider a function $g:\{1, \ldots, n+$ $1\} \rightarrow(0, \infty)$. Obviously, $f=-\ln$ is convex and continuous on $(0, \infty)$, so we may apply Jensen's inequality to obtain

$$
\begin{aligned}
\ln \left[\frac{1}{n}\left(\alpha \sum_{t=1}^{n} g(t)+(1-\alpha) \sum_{t=2}^{n+1} g(t)\right)\right] & =\ln \left[\frac{1}{n} \int_{1}^{n+1} g(t) \diamond_{\alpha} t\right] \\
& \geq \frac{1}{n} \int_{1}^{n+1} \ln (g(t)) \diamond_{\alpha} t \\
& =\frac{1}{n}\left[\alpha \sum_{t=1}^{n} \ln (g(t))+(1-\alpha) \sum_{t=2}^{n+1} \ln (g(t))\right] \\
& =\ln \left\{\prod_{t=1}^{n} g(t)\right\}^{\alpha / n}+\ln \left\{\prod_{t=2}^{n+1} g(t)\right\}^{(1-\alpha) / n},
\end{aligned}
$$

and hence

$$
\frac{1}{n}\left(\alpha \sum_{t=1}^{n} g(t)+(1-\alpha) \sum_{t=2}^{n+1} g(t)\right) \geq\left\{\prod_{t=1}^{n} g(t)\right\}^{\alpha / n}\left\{\prod_{t=2}^{n+1} g(t)\right\}^{(1-\alpha) / n}
$$

When $\alpha=1$, we obtain the well-known arithmetic-mean geometric-mean inequality:

$$
\frac{1}{n} \sum_{t=1}^{n} g(t) \geq\left\{\prod_{t=1}^{n} g(t)\right\}^{1 / n}
$$

When $\alpha=0$, we also have

$$
\frac{1}{n} \sum_{t=2}^{n+1} g(t) \geq\left\{\prod_{t=2}^{n+1} g(t)\right\}^{1 / n}
$$


Moulay Rchid Sidi Ammi et al.

(vi) Let $\mathbb{T}=2^{\mathbb{N}_{0}}$ and $N \in \mathbb{N}$. We can apply Theorem 3.3 with $a=1, b=2^{N}$, and $g:\left\{2^{k}\right.$ : $0 \leq k \leq N\} \rightarrow(0, \infty)$. Then we get

$$
\begin{aligned}
& \ln \left\{\frac{\int_{1}^{2^{N}} g(t) \diamond_{\alpha} t}{2^{N}-1}\right\}=\ln \left\{\alpha \frac{\int_{1}^{2^{N}} g(t) \Delta t}{2^{N}-1}+(1-\alpha) \frac{\int_{1}^{2^{N}} g(t) \nabla t}{2^{N}-1}\right\} \\
& =\ln \left\{\frac{\alpha \sum_{k=0}^{N-1} 2^{k} g\left(2^{k}\right)}{2^{N}-1}+\frac{(1-\alpha) \sum_{k=1}^{N} 2^{k} g\left(2^{k}\right)}{2^{N}-1}\right\} \\
& \geq \frac{\int_{1}^{2^{N}} \ln (g(t)) \nabla_{\alpha} t}{2^{N}-1} \\
& =\alpha \frac{\int_{1}^{2^{N}} \ln (g(t)) \Delta t}{2^{N}-1}+(1-\alpha) \frac{\int_{1}^{2^{N}} \ln (g(t)) \nabla t}{2^{N}-1} \\
& =\frac{\alpha \sum_{k=0}^{N-1} 2^{k} \ln \left(g\left(2^{k}\right)\right)}{2^{N}-1}+\frac{(1-\alpha) \sum_{k=1}^{N} 2^{k} \ln \left(g\left(2^{k}\right)\right)}{2^{N}-1} \\
& =\frac{\sum_{k=0}^{N-1} \ln \left(g\left(2^{k}\right)\right)^{\alpha 2^{k}}}{2^{N}-1}+\frac{\sum_{k=1}^{N} \ln \left(g\left(2^{k}\right)\right)(1-\alpha) 2^{k}}{2^{N}-1} \\
& =\frac{\ln \prod_{k=0}^{N-1}\left(g\left(2^{k}\right)\right)^{\alpha 2^{k}}}{2^{N}-1}+\frac{\ln \left(\prod_{k=1}^{N} g\left(2^{k}\right)\right)^{(1-\alpha) 2^{k}}}{2^{N}-1} \\
& =\ln \left\{\prod_{k=0}^{N-1}\left(g\left(2^{k}\right)\right)^{\alpha 2^{k}}\right\}^{1 /\left(2^{N}-1\right)}+\ln \left\{\prod_{k=1}^{N}\left(g\left(2^{k}\right)\right)^{(1-\alpha) 2^{k}}\right\}^{1 /\left(2^{N}-1\right)} \\
& =\ln \left(\left\{\prod_{k=0}^{N-1}\left(g\left(2^{k}\right)\right)^{\alpha 2^{k}}\right\}^{1 /\left(2^{N}-1\right)}\left\{\prod_{k=1}^{N}\left(g\left(2^{k}\right)\right)^{(1-\alpha) 2^{k}}\right\}^{1 /\left(2^{N}-1\right)}\right) .
\end{aligned}
$$

We conclude that

$$
\begin{aligned}
& \ln \left\{\frac{\alpha \sum_{k=0}^{N-1} 2^{k} g\left(2^{k}\right)+(1-\alpha) \sum_{k=1}^{N} 2^{k} g\left(2^{k}\right)}{2^{N}-1}\right\} \\
& \quad \geq \ln \left(\left\{\prod_{k=0}^{N-1}\left(g\left(2^{k}\right)\right)^{\alpha 2^{k}}\right\}^{1 /\left(2^{N}-1\right)}\left\{\prod_{k=1}^{N}\left(g\left(2^{k}\right)\right)^{(1-\alpha) 2^{k}}\right\}^{1 /\left(2^{N}-1\right)}\right) .
\end{aligned}
$$

On the other hand,

$$
\alpha \sum_{k=0}^{N-1} 2^{k} g\left(2^{k}\right)+(1-\alpha) \sum_{k=1}^{N} 2^{k} g\left(2^{k}\right)=\sum_{k=1}^{N-1} 2^{k} g\left(2^{k}\right)+\alpha g(1)+(1-\alpha) 2^{N} g\left(2^{N}\right) .
$$

It follows that

$$
\frac{\sum_{k=1}^{N-1} 2^{k} g\left(2^{k}\right)+\alpha g(1)+(1-\alpha) 2^{N} g\left(2^{N}\right)}{2^{N}-1} \geq\left\{\prod_{k=0}^{N-1}\left(g\left(2^{k}\right)\right)^{\alpha 2^{k}}\right\}^{1 /\left(2^{N}-1\right)}\left\{\prod_{k=1}^{N}\left(g\left(2^{k}\right)\right)^{(1-\alpha) 2^{k}}\right\}^{1 /\left(2^{N}-1\right)} .
$$


In the particular case when $\alpha=1$, we have

$$
\frac{\sum_{k=0}^{N-1} 2^{k} g\left(2^{k}\right)}{2^{N}-1} \geq\left\{\prod_{k=0}^{N-1}\left(g\left(2^{k}\right)\right)^{2^{k}}\right\}^{1 /\left(2^{N}-1\right)},
$$

and when $\alpha=0$ we get the inequality

$$
\frac{\sum_{k=1}^{N} 2^{k} g\left(2^{k}\right)}{2^{N}-1} \geq\left\{\prod_{k=1}^{N}\left(g\left(2^{k}\right)\right)^{2^{k}}\right\}^{1 /\left(2^{N}-1\right)} .
$$

\section{Related diamond- $\alpha$ integral inequalities}

The usual proof of Hölder's inequality use the basic Young inequality $x^{1 / p} y^{1 / q} \leq x / p+y / q$ for nonnegative $x$ and $y$. Here, we present a proof based on the application of Jensen's inequality (Theorem 3.3).

Theorem 4.1 (Hölder's inequality). Let $\mathbb{T}$ be a time scale, $a, b \in \mathbb{T}$ with $a<b$, and $f, g, h \in$ $C\left([a, b]_{\mathbb{T}},[0, \infty)\right)$ with $\int_{a}^{b} h(x) g^{q}(x) \diamond_{\alpha} x>0$, where $q$ is the Hölder conjugate number of $p$, that is, $1 / p+1 / q=1$ with $1<p$. Then we have

$$
\int_{a}^{b} h(x) f(x) g(x) \nabla_{\alpha} x \leq\left(\int_{a}^{b} h(x) f^{p}(x) \nabla_{\alpha} x\right)^{1 / p}\left(\int_{a}^{b} h(x) g^{q}(x) \triangleright_{\alpha} x\right)^{1 / q} .
$$

Proof. Choosing $f(x)=x^{p}$ in Theorem 3.3, which for $p>1$ is obviously a convex function on $[0, \infty)$, we have

$$
\left(\frac{\int_{a}^{b}|h(s)| g(s) \diamond_{\alpha} s}{\int_{a}^{b}|h(s)| \diamond_{\alpha} s}\right)^{p} \leq \frac{\int_{a}^{b}|h(s)|(g(s))^{p} \diamond_{\alpha} s}{\int_{a}^{b}|h(s)| \diamond_{\alpha} s} .
$$

Inequality (4.1) is trivially true in the case when $g$ is identically zero. We consider two cases: (i) $g(x)>0$ for all $x \in[a, b]_{\mathbb{T}}$; (ii) there exists at least one $x \in[a, b]_{\mathbb{T}}$ such that $g(x)=0$. We begin with situation (i). Replacing $g$ by $f g^{-q / p}$ and $|h(x)|$ by $h g^{q}$ in inequality (4.2), we get

$$
\left(\frac{\int_{a}^{b} h(x) g^{q}(x) f(x) g^{-q / p}(x) \diamond_{\alpha} x}{\int_{a}^{b} h(x) g^{q}(x) \diamond_{\alpha} x}\right)^{p} \leq \frac{\int_{a}^{b} h(x) g^{q}(x)\left(f(x) g^{-q / p}(x)\right)^{p} \diamond_{\alpha} x}{\int_{a}^{b} h(x) g^{q}(x) \diamond_{\alpha} x} .
$$

Using the fact that $1 / p+1 / q=1$, we obtain that

$$
\int_{a}^{b} h(x) f(x) g(x) \diamond_{\alpha} x \leq\left(\int_{a}^{b} h(x) f^{p}(x) \diamond_{\alpha} x\right)^{1 / p}\left(\int_{a}^{b} h(x) g^{q}(x) \nabla_{\alpha} x\right)^{1 / q} .
$$

We now consider situation (ii). Let $G=\left\{x \in[a, b]_{\mathbb{T}} \mid g(x)=0\right\}$. Then

$$
\begin{aligned}
\int_{a}^{b} h(x) f(x) g(x) \diamond_{\alpha} x & =\int_{[a, b]_{\mathbb{T}}-G} h(x) f(x) g(x) \diamond_{\alpha} x+\int_{G} h(x) f(x) g(x) \diamond_{\alpha} x \\
& =\int_{[a, b]_{\mathbb{T}}-G} h(x) f(x) g(x) \diamond_{\alpha} x
\end{aligned}
$$


because $\int_{G} h(x) f(x) g(x) \diamond_{\alpha} x=0$. For the set $[a, b]_{\mathbb{T}}-G$, we are in case (i), that is, $g(x)>0$, and it follows from (4.4) that

$$
\begin{aligned}
\int_{a}^{b} h(x) f(x) g(x) \diamond_{\alpha} x & =\int_{[a, b]_{\mathbb{T}}-G} h(x) f(x) g(x) \diamond_{\alpha} x \\
& \leq\left(\int_{[a, b]_{\mathbb{T}}-G} h(x) f^{p}(x) \diamond_{\alpha} x\right)^{1 / p}\left(\int_{[a, b]_{\mathbb{T}}-G} h(x) g^{q}(x) \diamond_{\alpha} x\right)^{1 / q} \\
& \leq\left(\int_{a}^{b} h(x) f^{p}(x) \diamond_{\alpha} x\right)^{1 / p}\left(\int_{a}^{b} h(x) g^{q}(x) \diamond_{\alpha} x\right)^{1 / q}
\end{aligned}
$$

Remark 4.2. In the particular case $h=1$, Theorem 4.1 gives the diamond- $\alpha$ version of classical Hölder's inequality:

$$
\int_{a}^{b}|f(x) g(x)| \nabla_{\alpha} x \leq\left(\int_{a}^{b}|f|^{p}(x) \diamond_{\alpha} x\right)^{1 / p}\left(\int_{a}^{b}|g|^{q}(x) \diamond_{\alpha} x\right)^{1 / q}
$$

where $p>1$ and $q=p /(p-1)$.

Remark 4.3. In the special case $p=q=2$, (4.1) reduces to the following diamond- $\alpha$ CauchySchwarz integral inequality on time scales:

$$
\int_{a}^{b}|f(x) g(x)| \nabla_{\alpha} x \leq \sqrt{\left(\int_{a}^{b} f^{2}(x) \diamond_{\alpha} x\right)\left(\int_{a}^{b} g^{2}(x) \diamond_{\alpha} x\right)} .
$$

We are now in position to prove a Minkowski inequality using our Hölder's inequality (4.1).

Theorem 4.4 (Minkowski's inequality). Let $\mathbb{T}$ be a time scale, $a, b \in \mathbb{T}$ with $a<b$, and $p>1$. For continuous functions $f, g:[a, b]_{\mathbb{T}} \rightarrow \mathbb{R}$, we have

$$
\left(\int_{a}^{b}|(f+g)(x)|^{p} \nabla_{\alpha} x\right)^{1 / p} \leq\left(\int_{a}^{b}|f(x)|^{p} \nabla_{\alpha} x\right)^{1 / p}+\left(\int_{a}^{b}|g(x)|^{p} \nabla_{\alpha} x\right)^{1 / p} .
$$

Proof. We have, by the triangle inequality, that

$$
\begin{aligned}
\int_{a}^{b}|f(x)+g(x)|^{p} \diamond_{\alpha} x & =\int_{a}^{b}|f(x)+g(x)|^{p-1}|f(x)+g(x)| \nabla_{\alpha} x \\
& \leq \int_{a}^{b}|f(x)||f(x)+g(x)|^{p-1} \nabla_{\alpha} x+\int_{a}^{b}|g(x)||f(x)+g(x)|^{p-1} \nabla_{\alpha} x .
\end{aligned}
$$


Applying now Hölder's inequality with $q=p /(p-1)$ to (4.10), we obtain

$$
\begin{aligned}
\int_{a}^{b}|f(x)+g(x)|^{p} \diamond_{\alpha} x \leq & {\left[\int_{a}^{b}|f(x)|^{p} \nabla_{\alpha} x\right]^{1 / p}\left[\int_{a}^{b}|f(x)+g(x)|^{(p-1) q} \nabla_{\alpha} x\right]^{1 / q} } \\
& +\left[\int_{a}^{b}|g(x)|^{p} \nabla_{\alpha} x\right]^{1 / p}\left[\int_{a}^{b}|f(x)+g(x)|^{(p-1) q} \nabla_{\alpha} x\right]^{1 / q} \\
= & \left\{\left[\int_{a}^{b}|f(x)|^{p} \diamond_{\alpha} x\right]^{1 / p}+\left[\int_{a}^{b}|g(x)|^{p} \diamond_{\alpha} x\right]^{1 / p}\right\}\left[\int_{a}^{b}|f(x)+g(x)|^{p} \diamond_{\alpha} x\right]^{1 / q} .
\end{aligned}
$$

Dividing both sides of the last inequality by

$$
\left[\int_{a}^{b}|f(x)+g(x)|^{p} \diamond_{\alpha} x\right]^{1 / q}
$$

we get the desired conclusion.

As another application of Theorem 3.3, we have Theorem 4.5, as follows.

Theorem 4.5. Let $\mathbb{T}$ be a time scale, $a, b \in \mathbb{T}$ with $a<b$, and $f, g, h \in C\left([a, b]_{\mathbb{T}},[0, \infty)\right)$.

(i) If $p>1$, then

$$
\left\{\left(\int_{a}^{b} h f \diamond_{\alpha} x\right)^{p}+\left(\int_{a}^{b} h g \triangleright_{\alpha} x\right)^{p}\right\}^{1 / p} \leq \int_{a}^{b} h\left(f^{p}+g^{p}\right)^{1 / p} \nabla_{\alpha} x
$$

(ii) If $0<p<1$, then

$$
\left\{\left(\int_{a}^{b} h f \diamond_{\alpha} x\right)^{p}+\left(\int_{a}^{b} h g \triangleright_{\alpha} x\right)^{p}\right\}^{1 / p} \geq \int_{a}^{b} h\left(f^{p}+g^{p}\right)^{1 / p} \nabla_{\alpha} x
$$

Proof. We prove only (i). The proof of (ii) is similar. Inequality (i) is trivially true when $f$ is zero: both the left and right hand sides reduce to $\int_{a}^{b} h g \diamond_{\alpha} x$. Otherwise, applying Theorem 3.3 with $f(x)=\left(1+x^{p}\right)^{1 / p}$, which is clearly convex on $(0, \infty)$, we obtain

$$
\left(1+\frac{\left(\int_{a}^{b} h f \diamond_{\alpha} x\right)^{p}}{\left(\int_{a}^{b} h \diamond_{\alpha} x\right)^{p}}\right)^{1 / p} \leq \frac{\int_{a}^{b} h\left(1+f^{p}\right)^{1 / p} \nabla_{\alpha} x}{\int_{a}^{b} h \diamond_{\alpha} x} .
$$

In other words,

$$
\left[\left(\int_{a}^{b} h \diamond_{\alpha} x\right)^{p}+\left(\int_{a}^{b} h f \diamond_{\alpha} x\right)^{p}\right]^{1 / p} \leq \int_{a}^{b} h\left(1+f^{p}\right)^{1 / p} \nabla_{\alpha} x .
$$

Changing $h$ and $f$ by $h f / \int_{a}^{b} h f \diamond_{\alpha} x$ and $g / f$ in the last inequality, respectively, we obtain directly the inequality (i) of Theorem 4.5. 


\section{Acknowledgments}

The authors were supported by the Portuguese Foundation for Science and Technology (FCT), through the Centre for Research on Optimization and Control (CEOC) of the University of Aveiro, cofinanced by the European Community Fund FEDER/POCI 2010 (all the three authors); the postdoc fellowship SFRH/BPD/20934/2004 (Sidi Ammi); the PhD fellowship SFRH/BD/39816/2007 (Ferreira); and the research project PTDC/MAT/72840/2006 (Torres). The authors are grateful to three referees and the editor assigned to handle the review process for several helpful comments and the careful reading of the manuscript.

\section{References}

[1] D. S. Mitrinović, Analytic Inequalities, Springer, New York, NY, USA, 1970.

[2] R. P. Agarwal, M. Bohner, and A. Peterson, "Inequalities on time scales: a survey," Mathematical Inequalities \& Applications, vol. 4, no. 4, pp. 535-557, 2001.

[3] U. M. Özkan, M. Z. Sarikaya, and H. Yildirim, "Extensions of certain integral inequalities on time scales," Applied Mathematics Letters. In press http://dx.doi.org/10.1016/j.aml.2007.06.008 .

[4] Q. Sheng, M. Fadag, J. Henderson, and J. M. Davis, "An exploration of combined dynamic derivatives on time scales and their applications," Nonlinear Analysis: Real World Applications, vol. 7, no. 3, pp. 395-413, 2006.

[5] U. M. Ozkan and H. Yildirim, "Steffensen's integral inequality on time scales," Journal of Inequalities and Applications, vol. 2007, Article ID 46524, 10 pages, 2007.

[6] Q. Sheng, "Hybrid approximations via second order combined dynamic derivatives on time scales," Electronic Journal of Qualitative Theory of Differential Equations, no. 17, pp. 1-13, 2007.

[7] S. Hilger, Ein maßkettenkalkül mit anwendung auf zentrumsmannigfaltigkeiten, Ph.D. thesis, Universität Würzburg, Würzburg, Germany, 1988.

[8] S. Hilger, "Analysis on measure chains-a unified approach to continuous and discrete calculus," Results in Mathematics, vol. 18, no. 1-2, pp. 18-56, 1990.

[9] S. Hilger, "Differential and difference calculus-unified!," Nonlinear Analysis: Theory, Methods $\mathcal{E}$ Applications, vol. 30, no. 5, pp. 2683-2694, 1997.

[10] R. P. Agarwal and M. Bohner, "Basic calculus on time scales and some of its applications," Results in Mathematics, vol. 35, no. 1-2, pp. 3-22, 1999.

[11] R. P. Agarwal, M. Bohner, D. O'Regan, and A. Peterson, "Dynamic equations on time scales: a survey," Journal of Computational and Applied Mathematics, vol. 141, no. 1-2, pp. 1-26, 2002.

[12] R. P. Agarwal, M. Bohner, and P. J. Y. Wong, "Sturm-Liouville eigenvalue problems on time scales," Applied Mathematics and Computation, vol. 99, no. 2-3, pp. 153-166, 1999.

[13] R. P. Agarwal and D. O'Regan, "Nonlinear boundary value problems on time scales," Nonlinear Analysis: Theory, Methods E Applications, vol. 44, no. 4, pp. 527-535, 2001.

[14] F. M. Atici and G. Sh. Guseinov, "On Green's functions and positive solutions for boundary value problems on time scales," Journal of Computational and Applied Mathematics, vol. 141, no. 1-2, pp. 75-99, 2002.

[15] M. Bohner and A. Peterson, Dynamic Equations on Time Scales, Birkhäuser, Boston, Mass, USA, 2001.

[16] M. Bohner and A. Peterson, Eds., Advances in Dynamic Equations on Time Scales, Birkhäuser, Boston, Mass, USA, 2003.

[17] J. W. Rogers Jr. and Q. Sheng, "Notes on the diamond- $\alpha$ dynamic derivative on time scales," Journal of Mathematical Analysis and Applications, vol. 326, no. 1, pp. 228-241, 2007.

[18] G. B. Folland, Real Analysis, Pure and Applied Mathematics, John Wiley \& Sons, New York, NY, USA, 2nd edition, 1999.

[19] F.-H. Wong, C.-C. Yeh, and W.-C. Lian, "An extension of Jensen's inequality on time scales," Advances in Dynamical Systems and Applications, vol. 1, no. 1, pp. 113-120, 2006. 